\title{
Feeling-of-knowing experiences breed curiosity
}

Gregory Brooks ${ }^{\mathrm{ab} *}$, Haopei Yang ${ }^{\mathrm{ab}}$, and Stefan Köhler ${ }^{\mathrm{acd}}$

${ }^{a}$ The Brain and Mind Institute, University of Western Ontario, London, Canada; ${ }^{b}$ Graduate Program in Neuroscience, University of Western Ontario, London, Canada; ${ }^{c}$ Department of Psychology, University of Western Ontario, London, Canada; ${ }^{d}$ Rotman Research Institute, Baycrest Centre, Toronto, Canada

For correspondence: Gregory Brooks gbrook5@uwo.ca or Stefan Köhler stefank@uwo.ca The Brain and Mind Institute, Western Interdisciplinary Research Building, University of Western Ontario, London, ON N6A 5B7, Canada

Word Count (incl. tables, figure captions and title page): 12291 


\section{Feeling-of-knowing experiences breed curiosity}

A central tenet in theoretical work on metacognition is that retrieval experiences during memory search can exert control over behaviour. States of curiosity, which reflect motivational tendencies to seek out information, may play a critical role in this control function. We conducted two experiments to address this idea, focusing on links between feeling-of knowing (FOK) experiences, memory-search duration, and subsequent information-seeking behaviour. We administered an episodic FOK paradigm that probed memory for previously studied face-name pairs and subsequently provided an opportunity to select limited pairs for restudy. This set-up allowed us to test whether current search duration and subsequent restudy choices are biased towards information with high FOK ratings. Results revealed a positive relationship between FOK ratings and the response times of these judgements. We observed a similar positive relationship between FOK ratings and subsequent item selection for restudy. Moreover, experimental manipulations of FOK ratings based on familiarity of the face cues also had parallel effects. Our findings suggest that metacognitive experiences during unsuccessful retrieval from episodic memory can induce states of curiosity that shape behaviour beyond the immediate retrieval context. Curiosity may act as a bond to ensure that memory gaps identified through unsuccessful retrieval adaptively guide future learning.

Keywords: metacognition; information-seeking; retrieval; metamemory; familiarity

\section{Introduction}

Curiosity has become an increasingly studied topic for cognitive psychologists and neuroscientists alike. Contemporary definitions of curiosity emphasise motivational components and suggest that it is a cognitive state characterised by the desire to obtain information (Kidd \& Hayden, 2015; Gottlieb et al., 2016). It has been proposed to be distinct from other forms of information-seeking by virtue of being intrinsically motivated and independent of external reward (Loewenstein, 1994; Kidd \& Hayden, 2015; Gottlieb et al., 2016; Metcalfe et al., 2020).

With the surge in research on curiosity, the close link it shares with learning has become 
a central topic of investigation. Multiple studies have shown that memory encoding, as well as subsequent consolidation processes, are enhanced when an individual is in a state of curiosity. For example, in several studies it has been reported that trivia facts are remembered more accurately when corresponding questions (e.g. "what does the term dinosaur mean?") are rated as sparking curiosity, before the facts (e.g. "a terrible lizard”) are revealed (Gruber et al., 2014). These beneficial effects of curiosity on subsequent memory accuracy have been found to be lasting over delays of several days (McGillivray et al., 2015; Marvin \& Shohamy, 2016; Fastrich et al., 2018; Stare et al., 2018). Additionally, curiosity-dependent memory enhancements have been shown to extend to unrelated information presented coincidentally with the curiositytriggering trivia questions (Gruber et al., 2014). At the mechanistic level, there is evidence to suggest that curiosity-dependent effects on learning build on the engagement of reward circuitry, including the substantia nigra/ventral tegmental area (SN/VTA) and the ventral striatum (Kang et al., 2009; Gruber et al., 2014). In line with this empirical evidence is the theoretical suggestion that information may in and of itself be a reward. From this perspective, curiosity functions like other reward-driven behaviours, such as hunger or thirst, with information acting as the soughtafter reward (Marvin \& Shohamy, 2016). An important question to ask in this theoretical context is whether retrieval-related processes can induce a state of curiosity in which information that is inaccessible is experienced as rewarding, and therefore leads to subsequent information-seeking behaviour.

In research on metacognition it has been well-established that unsuccessful retrieval can be experienced in different ways, and that such experiences have behavioural relevance. A wellstudied example of an experience unique to memory retrieval is the tip-of-the-tongue (TOT) phenomenon (Brown \& McNeill, 1966). Another example is the feeling that we might be able to 
recognise the answer that we cannot recall among multiple alternatives, an experience that is referred to as a feeling-of-knowing (FOK) in the memory literature (Hart, 1965). Such FOK states have been documented in relation to retrieval of semantic information (e.g. "What is the capital city of Ghana?"), as well as retrieval of information from episodic memory (e.g. "What is the name of the boisterous individual I encountered at the party last night?"). Both TOT and FOK experiences have been suggested to guide decisions about when to stop memory search in situations that are characterised by a lack of recall success (e.g. Schwartz, 2001; Singer \& Tiede, 2008). Critically, it has also been proposed that they may shape subsequent decisions about whether to seek out the information that could not be recalled (Litman et al., 2005, Metcalfe, et al., 2017, Hanczakowski et al., 2014). For example, when a familiar person whose name we cannot recall is a celebrity, we may decide to Google the answer based on the context in which the person was encountered (e.g. a movie). This illustration highlights a potential role for metacognitive experiences during unsuccessful retrieval in motivating the type of informationseeking behaviour that defines curiosity, both during and after memory search. In the present study we aim to address this possibility by examining the relationship between metacognitive retrieval experiences, specifically FOKs, the duration of immediate memory search, and subsequent information-seeking behaviour.

Theoretical approaches to metamemory have made an important distinction between its monitoring and control functions (see Nelson, 1996; Koriat, 2007; and Moulin \& Souchay, 2014 for review). Nelson and colleagues have also elaborated in their work on how metacognitive monitoring and control functions may interact in iterative cycles (Nelson \& Leonesio, 1988; Nelson \& Narens, 1990, 1996; Nelson 1996). It has been argued in several theoretical models that these distinct functions of metamemory play a role in the acquisition, retention, and retrieval 
of information (Nelson 1996, Koriat, 2007). In the context of retrieval, the monitoring aspect encompasses processes related to assessing the progress and outcome of memory search. Metacognitive control, by contrast, pertains to how the experiences that emerge during monitoring guide behavioural choices during and following memory search.

Empirical research on metamemory has focused mostly on the monitoring aspect. Recent work has provided convincing evidence supporting the notion that FOKs are based on heuristic inferences, i.e. on inferences that rest on clues other than the sought-after information itself. Specifically, it has been observed that two main heuristic cues inform people's monitoringrelated judgements of metamemory: cue familiarity and target accessibility. Studies have shown that when cues are made to be more familiar, typically by priming prior to study, subsequent FOK ratings are higher relative to unprimed cues (Reder, 1987; Reder \& Ritter, 1992, Schwartz \& Metcalfe, 1992; Metcalfe et al., 1993). Related work has demonstrated that FOKs increase as the amount of recalled partial or associated information about a target and the ease with which this information comes to mind increases, a variable termed target accessibility (Koriat \& LevySadot, 2001). Such information could include, for example, remembering that someone's name begins with "M" or that it was similar to an actor's name. In episodic FOK studies, metacognitive judgements have also been shown to be influenced by retrieval of associated contextual information (see Thomas et al., 2011, Schwartz, et al., 2014; Hosey et al., 2009; Isingrini et al., 2016). It should be noted that heuristic inferences based on cue familiarity and target accessibility have been proposed to contribute to the formation of FOK experiences in conjunction (Koriat, 2007).

Decisions about the termination of memory search and about subsequent informationseeking speak to the control function of metacognitive retrieval experiences, which, to date, have 
been less frequently studied. One domain in which control functions of metacognitive retrieval experiences have been studied concerns the duration of unsuccessful memory search. The outcome from numerous studies converges on the finding that these variables are positively correlated. Of most relevance for the current research are studies that revealed this relationship in FOK paradigms (but see e.g. Schwartz, 2001 for similar results in research on TOT). These studies have typically focused on the relationship between FOK experiences and memory search during retrieval of semantic information. Gruneberg et al. (1977) first demonstrated that response times for the report of unsuccessful recall were longer for items for which participants indicated the presence of an FOK experience relative to items where such an experience was absent. Subsequent work also revealed that this relationship holds when a graded scale is used to probe for FOK experiences (Costermans et al., 1992). In other research on this topic, Nelson and colleagues showed that even response times for incorrect answers in response to factual questions were positively correlated with the strength of FOK experiences (Nelson \& Narens, 1980; Nelson et al., 1984). Although it is difficult to disentangle cause and effect in the relationship between the duration of memory search and FOKs (see Metcalfe, 2009), the findings reviewed are compatible with the view that FOK experiences exert control on behaviour at the level of gating the extent of memory search.

In discussions of the functional role of metacognitive retrieval experiences it has also been suggested that they may contribute to the control of behaviour outside of the context of the memory judgment at hand (Koriat, 2007). One behavioural domain in which their control functions may play out is in guiding subsequent information-seeking behaviour in the external environment as a reflection of curiosity. Although this idea has intuitive appeal, extant research that speaks to it directly is limited. To our knowledge, only a single study (Hanczakowski et al., 
2014) has explored this topic in relation to FOK experiences (but see Litman et al., 2005 and Metcalfe et al., 2017 for studies investigating it in relation to TOT experiences). This study focused on restudy-choices that immediately followed FOK judgments in an episodic-memory paradigm for arbitrary paired words that had been encountered in an initial study phase. Results showed that participants' restudy choices were correlated with FOK judgement on an item-byitem basis, such that items with unsuccessful recall of the associate and higher FOK ratings were selected for restudy more frequently than those with lower ratings. This finding suggests that the control function of FOKs may indeed include information-seeking behaviour. Given that behavioural choices directly followed the memory judgments on an item-by-item basis in this study, however, its results do not speak to whether information-seeking is influenced by prior FOK experiences in lasting ways, namely when the behavioural decision is made outside of the context of an immediately preceding memory search. Moreover, it also does not address any potential relationship between control functions of FOK that pertain to duration of internal memory search and those that pertain to information-seeking behaviour in the external environment. To the extent that memory search can also be considered to be a type of information-seeking behaviour it is possible that both control functions rely on shared mechanisms related to curiosity. This rationale builds on the notion that objective measures of information-seeking behaviour can serve as direct markers of state curiosity, an assertion that finds acceptance in the wider literature on curiosity (e.g. Hsee and Ruan, 2016; Lau et al., 2020; Oosterwijk et al., 2020; see Gruber \& Ranganath, 2019, for review and discussion).

In order to answer these questions we adopted a behavioural paradigm previously employed in our research on experiential aspects of episodic FOKs (Fiacconi et al., 2017). This paradigm makes use of face-name pairs, rather than word-pairs, to assess FOKs. This is 
important as these stimuli have more ecological validity than word-pairs, as FOK experiences, and familiarity in general, are frequently triggered in our everyday lives by the faces or names of people. In our paradigm, participants were tasked with attempting to recall a target name that had been paired with the image of a person's face in an initial memorisation phase, after which they were asked to provide an FOK judgement. Once they had completed this FOK test phase for each face-name pair, participants were exposed to the face cues once again, and were given the opportunity to seek out a limited number of the associated names for restudy. We also included a manipulation of cue familiarity, given that it has been shown to be one of the sources for the heuristic inferential processes that give rise to FOKs (Reder, 1987; Reder \& Ritter, 1992, Schwartz \& Metcalfe, 1992; Metcalfe et al., 1993). In Experiment 1, we assessed the relationship of FOK experiences to the control of behaviour employing previously studied and novel face stimuli during the FOK test phase. We offered unlimited time for recall so as to evaluate the relationship between FOKs and response times at the time of a retrieval attempt, as well as between FOKs and subsequent information-seeking behaviour. In Experiment 2, we only employed previously studied faces in the FOK test phase but included a priming manipulation to selectively probe for the role of cue (i.e. face) familiarity in the relationship between metacognitive retrieval experiences and subsequent information-seeking behaviour.

We anticipated to find that the strength of FOK experiences would not only be correlated with participants' response times for corresponding judgments, but that they would also predict participants' subsequent information-seeking when offered opportunities for restudy. The manipulation of cue familiarity in our experimental paradigm also allowed us to explore a potential causal role for FOK experiences in these relationships. Specifically, we predicted that the well documented boost of FOKs from increased cue familiarity would lead to corresponding 
increases in immediate memory-search duration and in subsequent information-seeking behaviour.

\section{Experiment I}

\section{Methods and Materials}

\section{Participants}

In Experiment 1, 45 undergraduate and graduate student participants were recruited from Western University to take part in the study in exchange for monetary compensation. The data of 36 participants (26 female; age range 18-25) were included in our final analyses, with the remaining 9 excluded due to insufficient distribution of FOK values (i.e. less than 5 instances for 2 of the 5 scale values on unsuccessful recall trials). This exclusion criterion was introduced to ensure a sufficient number of trials in each rating for correlation analyses. All experimental procedures were approved by the Non-Medical Research Ethics Board at the University of Western Ontario.

\section{Materials}

All face stimuli used in this paradigm were taken from the Chicago Face Database (Ma et al., 2015) and were screened using the published norming data to ensure uniformity in terms of neutral emotional expression and perceived attractiveness. Selection criteria included a rating below 3.5 (on a 7-point scale) on all emotional expressions (afraid, angry, happy, sad, surprised, disgusted, and threatening), and attractiveness ratings between 2 and 5 on the 7-point scale. Of the faces that met these criteria, a total of 78 faces were randomly selected for experimental use. 
For this study, 156 English names were selected from the U.S. Census Bureau 1990 (https://catalog.data.gov/dataset/names-from-census-1990) for use in the study and recognition phases of the experiment. The total set was composed of 78 male first names, 78 female first names, and 156 surnames of medium frequency in the population (frequency rates between $0.15 \%$ and $.5 \%$ for first names, and between $0.05 \%$ and $0.5 \%$ for surnames, respectively). Explicit efforts were made to avoid any overlap in pronunciation or spelling between the names selected (e.g. Julie and Julia or Robert and Roberts), and to avoid any reference to celebrities. First and last names were then paired to create 156 different full names of comparable length (11 to 17 characters; $M=12.9, S D=1$ ), and comparable syllable count (3 to 5).

For the purpose of counterbalancing, 78 faces were paired with two sets of names, with each participant assigned to one set. Assignment of names to faces was pseudo-random, with the restriction that sex be matched. The remaining non-assigned 78 names served as novel lures in the forced choice recognition memory test. Of the 78 matched face-name pairs, 52 were randomly assigned to be memorised (20 Caucasian females, 20 Caucasian males, 6 AfricanAmerican females and 6 African-American males), and the remaining 26 (10 Caucasian males, 10 Caucasian females, 3 African-American males and 3 African-American females) were used as novel stimuli in the FOK test phase.

\section{Procedure}

The experiment was administered using Psychophysics Toolbox Version-3 (http://www.psychtoolbox.org/) and MATLAB R2018b (The MathWorks, Natick, MA) with a 14-inch laptop. It included four different phases (Figure 1), taking approximately 35 minutes for completion. 
In the first part, participants were asked to memorise a set of 52 face-name pairs. Each pair appeared on the screen for $3 \mathrm{~s}$ with the face appearing above the name. Following a $500 \mathrm{~ms}$ interstimulus interval (ISI), the next pair was presented. Participants were offered a break halfway through this study phase.

The second phase served for memory testing and began immediately after completion of the study phase. Here, participants saw the 52 previously studied faces, along with 26 novel ones, for an unlimited duration, and they were instructed to try and recall the name associated with each face. On each trial, they responded to two self-paced memory judgement prompts. The first judgment required a yes/no response concerning the perceived success of their attempted name recall. The second judgement required FOK ratings; participants were asked to estimate the likelihood that they would be able to recognise the name associated with the face prompt, if provided, on a 5-point Likert scale (from 1/very unlikely to 5/very likely). As per the suggestion of Koriat (1993), this judgement was elicited for all faces presented, regardless of the participants' indication of perceived success on any given trial. Following these two judgements, the next face would appear on the screen after a $500 \mathrm{~ms}$ ISI.

After the FOK test phase was completed, participants entered the restudy phase. Here they were given an opportunity to select up to 39 of the 78 faces previously used as prompts in the FOK test phase for exposure to the associated name. The criterion of allowing restudy of half of the face-name pairs was selected as similar studies using restudy choices found a limitation of such choices to $50 \%$ of items to offer a sensitive manipulation to probe information-seeking (i.e. DeCaro \& Thomas, 2019). Note that, unbeknownst to participants, 52 of the 78 faces would have been memorised initially, with the other 26 only having served as lures in the FOK test phase. Thus, this exposure constituted a restudy or a first study opportunity, respectively. If the 
participant chose to see the name for a given prompt, the face-name pair would appear on the screen for $3 \mathrm{~s}$. After this interval, or if they chose not to see the name, the next face would appear, following a $500 \mathrm{~ms}$ ISI. Throughout this phase, participants were also exposed, in the corner of the screen, to a countdown of how many more face-name pairs were still available for exposure. If the participant reached the maximum of 39 possible exposures, they were forced to respond 'no' to the restudy prompt for the remainder of trials. No explicit mention of an additional future memory test was made before or during the restudy phase.

In the fourth and last phase of the experiment, participants completed a self-paced forcedchoice recognition test for the names of all 78 faces used in the FOK test phase, which could constitute faces initially memorised as well as faces employed as lures, regardless of whether they had been selected for exposure in the restudy phase or not. In this recognition test, three name options were presented for each face, namely the name corresponding to the face, a previously seen name that belonged to one of the other previously studied faces, and an entirely novel name. The three choices were matched for sex and were presented randomly in one of three positions.

\section{Results}

Do FOK ratings show validity in the current experimental paradigm?

In our first analysis we examined whether the FOK ratings obtained in our experiment carried validity by virtue of being sensitive to the study manipulation. This analysis leveraged the fact that not all faces for which FOK ratings were obtained had been studied during the memorisation of face-name pairs. In line with other studies on FOK experiences (i.e. Fiacconi et al., 2017), this analysis was limited to trials in which participants indicated that recall was unsuccessful. As 
expected, the average FOK ratings were significantly higher for previously studied than for novel face cues (see Table 1 for mean FOK ratings), $t(36)=10.11, p<0.001, d=1.68$. Note that, although we included a self-report measure of recall success rather than an opportunity to type out the response as typically done (i.e. Schwartz \& Metcalfe, 1992), the trials with self-reported success had numerically higher accuracy in the final recognition-memory test than trials on which recall was perceived to be unsuccessful (see Table 1), supporting our conjecture that this self-report does indeed capture aspects of recall success. However, inferential statistics could not be performed for this comparison, given the small number of trials on which participants indicated perceived recall success, combined with exclusion of items that were restudied prior to the recognition-memory test.

A second way to confirm the validity of FOK ratings is to show that they have predictive value for subsequent accuracy in recognition-memory judgments of names, despite following trials without successful recall. Towards this end, we computed gamma correlations for individual participants between their FOK ratings and performance on the recognition memory test (Nelson, 1984). Importantly, in order to control for any influence of repeated study, this calculation was completed only, not only for trials without successful recall, but also for trials in which names had not been selected for restudy. The average gamma correlation between FOK rating and recognition memory performance for all trials $(M=0.14, S D=0.37)$ was significantly greater than zero, $t(34)=2.22, p=0.033, d=0.38$. This significant relationship was also present when only initially studied face name-pairs were considered in the correlation $(M=0.19, S D=$ $0.35), t(34)=3.18, p=0.003, d=0.54$. These results provide further support for the validity of the FOK ratings provided by participants. 
Are FOK ratings related to response times during attempts to recall the names corresponding to face cues?

The first marker of curiosity that we examined was that of response times for the initial recall attempts. Specifically, to calculate response times, we focused on the duration of the recall attempt that participants engaged in for each trial in the FOK test phase. To assess the relationship between FOK ratings and response times (Figure 2A) we calculated Spearman correlations for each participant, between values on both dimensions. We found a positive correlation when all trials were included (Mean $r h o=0.34, S D=0.21)$, but also when trials without perceived successful recall were excluded (Mean rho $=0.34, S D=0.20$; note that in the large majority of trials, recall was perceived to be unsuccessful, as evident in Table 1). In both cases, the mean Spearman correlation was found to be larger than zero, $t(35)=9.78, p<0.001, d$ $=1.63$ and $t(35)=10.25, p<0.001, d=1.71$, respectively (Figure $2 \mathbf{B}$ ). Next, to compare the average response times for the memory judgements for unsuccessful recall trials between face cues that had previously been encountered and those that were novel, we conducted a $2 \times 2$ ANOVA, with restudied versus not restudied as the second factor. We found a significant main effect related to whether the face cue had been previously encountered, such that the initially studied trials $(M=3420 \mathrm{~ms}, S D=2048 \mathrm{~ms})$ had longer response times than trials with novel face cues $(M=3038 \mathrm{~ms}, S D=1959 \mathrm{~ms}), F(1,35)=6.86, p=0.006$. The interaction term did not reach statistical significance, $F(1,35)=0.25, p=0.62$. Taken together, these results suggest, in line with prior findings (e.g. Costermans et al., 1992), that the duration of search during memory judgments is related to the resulting FOK ratings, and is affected by prior exposure to the cues and the information that is to be recalled. 
Is the impact of prior exposure on response times during recall attempts tied to FOK ratings?

While the analyses just summarised suggest that response times for search during memory judgements are related to prior exposure of the face cues, they do not provide an indication as to whether this relationship is tied to FOK ratings or independent. To address this question, we conducted a generalised mixed-effects model procedure on response times in R (R Core Team, 2013).

The selected model we used for our analysis contained fixed effect terms representing FOK rating, item status (whether it had been initially studied or not) and the interaction between these variables. Details about the development of this model, such as the trimming of nonsignificant effects, and the random effect terms included in it, are described in the Supplemental Information Section. Importantly, as we were particularly interested in situations in which recall was ultimately unsuccessful (and where there was no natural endpoint to memory search), trials with perceived success during recall in the FOK test phase were excluded from this analysis.

With this modelling, we found that participants' FOK ratings positively predicted the response time during the recall attempt (see Table 2). This relationship was equal for both items that were initially studied and those that were not, as evident by the non-significant interaction between FOK rating and item status. Critically, the non-significant effect of item status indicated that there was no contribution of prior cue exposure on response times that was independent of FOK ratings.

\section{Are FOK ratings related to subsequent information-seeking?}

As a marker of information-seeking, we focused on participants' choices to study select facenames pairs in the restudy phase that followed the FOK test phase. In this part, participants were 
given an opportunity to select a limited number of face-name pairs when provided with faces as cues. The corresponding names had either been memorised initially during the study phase or had not been encountered yet (in the case of faces that were novel in the FOK test phase). Our primary interest was to determine whether these choices in information-seeking behaviour could be predicted by the ratings provided in the FOK test phase, and whether they were affected by prior study. If the relationship between FOK experiences and information-seeking, depicted visually in Figure 3A, extends beyond the time of a recall attempt, as we hypothesised, then gamma correlation coefficients between FOK experiences and information-seeking choices should be positive, paralleling the relationship observed between FOK ratings and memory search time. We found that the mean gamma between FOK and restudy choices for all trials $(M=$ $0.27, S D=0.40)$ was significantly greater than zero, $t(35)=4.13, p<0.001, d=0.69$. When the correlation was performed only for trials without successful perceived recall $(M=0.26, S D=$ 0.37 ) the relationship remained significantly positive, $t(35)=4.24, p<0.001, d=0.71$ (Figure 3B). This significant positive relationship indicates that high FOK experiences are associated with higher subsequent engagement of information-seeking behaviour towards the information that could not be recalled than low FOK experiences.

Next, we asked whether information-seeking in the restudy phase was affected by whether the information that could not be recalled in the FOK test phase had in fact been studied previously (i.e. whether the cues were familiar or not). To address this question, we compared the proportion of initially studied pairs selected for restudy to the proportion of novel pairs selected for study. This comparison, when performed for all trials, revealed that previously studied face-name pairs were selected for restudy at a significantly greater rate than novel pairs (see Table 1 for proportions), $t(35)=2.83, p=0.008, d=0.47$. This difference remained 
significant when the comparison was restricted to trials in which prior recall of names was perceived to be unsuccessful (see Table 1 for proportions), $t(35)=2.81, p=0.008, d=0.47$. These findings support our hypothesis that FOK ratings are related to the information that participants subsequently choose to seek. Moreover, they suggest that pertinent choices are impacted by the familiarity of the cues, biasing behavioural choices towards previously studied information. Overall, these results highlight parallels in the relationship between FOK experiences and search behaviour during memory retrieval, and that between FOK experiences and subsequent information-seeking behaviour.

Is the impact of prior exposure to face cues on subsequent information-seeking tied to FOK ratings?

As in our analyses of response time data, the analyses focusing on the relationship between prior exposure and subsequent restudy choices do not provide information as to whether this effect is tied to FOK ratings or independent. To address this question, we conducted another mixedeffects modelling analysis, similar to the one performed with response times. As before, only trials in which recall was perceived to be unsuccessful were included.

The selected model contained fixed effect terms representing item familiarity, FOK rating and the interaction between these factors (see Supplemental Information Section for further methodological detail on model selection). This mixed-effects model revealed that participants' FOK rating positively predicted subsequent restudy choices for items that were initially studied but not those encountered for the first time during the FOK test phase (see Table 3). For previously studied items, the log-odds of restudying the name associated with a face cue increased by 0.36 for each rating point on the 5-point FOK rating scale. In simpler terms, this means that for a face cue that was rated one point higher on the FOK rating scale than another 
item, the odds of restudying the higher-rated cue were 1.43 times as large. Critically, the effect of item status was non-significant in these analyses, indicating that there was no contribution of prior cue exposure on subsequent information-seeking behaviour that was independent of FOK ratings.

Is information-seeking related to response times during prior recall attempts?

Inasmuch as our results point to a link between FOK ratings and response times during the recall attempt, as well as between FOK ratings and subsequent information-seeking behaviour, an important question that remains to be answered is whether participants showed an increased tendency towards studying items for which they spent more time searching for an answer. This was explored through the $2 \times 2$ ANOVA that we also mentioned when assessing the influence of prior study on memory search times. A significant main effect of restudy was revealed, both when the ANOVA was conducted on all trials $F(1,35)=5.84, p=0.02$ and when it was restricted to trials without perceived recall success $F(1,35)=6.86, p=0.01$. More specifically, trials later chosen for restudy $(M=3357 \mathrm{~ms}, S D=1997 \mathrm{~ms}$ for all trials; $M=3367 \mathrm{~ms}, S D=$ $2037 \mathrm{~ms}$ for trials without recall success) had significantly longer response times for the previous recall attempt than those that were not chosen $(M=3098 \mathrm{~ms}, S D=1881 \mathrm{~ms}$ for all trials; $M=$ $3091 \mathrm{~ms}, S D=1942 \mathrm{~ms}$ for trials without recall success). No significant interaction between initial study status and restudy decision on recall response time was found for either ANOVA, $F(1,35)=0.33, p=0.57$ and $F(1,35)=0.25, p=0.62$, indicating that the observed difference in response time between restudied and not restudied items was equal for previously studied and novel face cues. This pattern of results suggests that the mechanisms through which FOKs shape immediate memory search and those through which they guide subsequent information-seeking may be overlapping. 


\section{Experiment II}

We conducted a second experiment with two main goals in mind. Our first goal was to replicate the predictive relationship between FOK ratings and subsequent information-seeking we observed in Experiment 1. Our second goal was to assess the impact of cue familiarity on the relationship between FOK ratings and subsequent information-seeking behaviour in a more selective manner. In Experiment 1 we manipulated whether items encountered during the FOK test phase had previously been studied in association with corresponding names or not. As such the behavioural differences we observed in relation to this manipulation could be due to prior exposure to the face cues, the memorisation of corresponding names, or a combination of these two factors. A consideration of the role of cue familiarity in and of itself is important in the context of our focus on motivational aspects of FOK experiences because an extensive literature suggests that this familiarity can serve as one of the sources for the inferential heuristic process that has been proposed to underlie FOK judgments (see Schwartz \& Metcalfe, 1992; Koriat \& Levy-Sadot, 2001).

\section{Methods and Materials}

\section{Participants}

Thirty-three English-speaking undergraduate participants from Western University took part in Experiment 2 in exchange for course credit. The data of 29 participants ( 15 female; age range 17-22) were used in all analyses, with the remaining 4 participants being excluded due to an insufficient distribution of FOK values across the scale (see exclusion criterion from Experiment 1). Again, all experimental procedures were approved by the Non-Medical Research Ethics Board at the University of Western Ontario. 


\section{Materials}

The same set of 78 face stimuli from Experiment 1 was used in Experiment 2, along with 52 additional faces that still met the criteria detailed for Experiment 1. Once again, two sets of pseudo-randomly matched face-name pairs were created. In each set, 78 face-name pairs (30 Caucasian males, 30 Caucasian females, 9 African-American males and 9 African-American females) were randomly selected to be studied in the study phase. The remaining 78 unmatched names served as novel lures in the forced-choice recognition test. Of the 78 faces to be memorised, 26 were chosen to be primed in the priming phase (a third of each demographic). The priming phase also featured the remaining 52 unpaired faces as distractors (20 Caucasian males, 20 Caucasian females, 6 African-American males, 6 African-American females). Note that the rationale for this counterbalancing parallels that employed in Experiment 1. Participants were randomly assigned to one of the two stimuli sets prior to beginning the experiment.

\section{Procedure}

The behavioural paradigm employed was very similar to the one used in Experiment 1. This time, however, the paradigm, which was administered on a 15.6-inch laptop, proceeded through five different phases and took approximately 45 minutes to complete (Figure 4). In the added first phase (i.e. the priming phase) participants were exposed to 26 of the faces that would later reappear in the study phase, alongside 52 distractor faces. During this self-paced part, participants were instructed to rate the likeability of the person in each image on a 5-point scale. The phase structure of the remaining parts of Experiment 2 was identical to that in Experiment 1, including a study phase, an FOK test phase, a restudy phase, and a final forced-choice recognition test. 
The procedural details of phases two to five were identical to those in Experiment 1 except for the inclusion of primed and unprimed items in the study phase (78 face-name pairs, 26 being primed), and two modifications in the FOK test phase. One modification was related to the composition of the list of face cues. Instead of being presented with previously studied and nonstudied face cues (i.e. our manipulation in Experiment 1), participants were only exposed to faces that had previously been studied, with a third of items having been primed prior to study. A second modification concerned the timing of trials in the FOK test phase. Specifically, participants were exposed to each face cue for $3 \mathrm{~s}$, rather than for an unlimited duration, before being directed to the subsequent memory-judgement prompts.

\section{Results}

Are FOK ratings and final recognition-memory judgments sensitive to the manipulation of familiarity of the face cues through priming?

In the first analyses for this experiment, we compared the FOK ratings and subsequent forcedchoice recognition-memory performance for primed faces with those for unprimed faces, to ensure that our priming manipulation had the expected effects. Like in Experiment 1, the trials in which participants perceived recall to be successful had numerically higher recognition-memory accuracies than trials where this recall was perceived as unsuccessful (see Table 1). As expected, average FOK ratings for primed cues that were not successfully recalled were significantly greater than average FOK ratings for unprimed cues that were not successfully recalled (see Table 1 for mean FOK ratings), $t(28)=6.28, p<0.001, d=1.17$. Also as expected, forcedchoice recognition-memory accuracy for primed faces was no different than the accuracy for unprimed faces when initial recall was unsuccessful (see Table 1 for recognition memory 
accuracies $), t(28)=-0.80, p=0.43, d=-0.15$. Like in Experiment 1, this comparison only considered pairs that were not selected for restudy (see Table 1 for proportion of primed and unprimed trials that were not restudied) to ensure that participants had equal exposure to the primed and unprimed face-name pairs. This pattern is in line with the basic notion that priming of face cues, without concurrent presentation of associated names, increases the familiarity of the face cue, which in turn inflates FOK ratings, but does not provide additional information for subsequent recognition of face name pairs. In other words, these findings confirm that our priming procedure was successful in manipulating familiarity as a cue that 'drives' FOK ratings (Reder, 1987; Reder \& Ritter, 1992, Schwartz \& Metcalfe, 1992; Metcalfe et al., 1993; Koriat \& Levy-Sadot, 2001).

\section{Do FOK ratings show validity in the current experimental paradigm?}

If participants' FOK ratings hold predictive validity, they should be related to future memory performance, as they were in Experiment 1. Again, we computed a gamma correlation coefficient, for each participant, between FOK ratings and subsequent recognition-memory accuracy, focusing only trials that were not selected for restudy. As expected, and as observed in Experiment 1, we found that the mean of these gamma correlations $(M=0.17, S D=0.27)$ was significantly greater than zero, $t(28)=3.48, p=0.0017, d=0.65$. When this analysis was repeated using only non-restudied trials that were also unsuccessfully recalled, the mean gamma correlation $(M=0.11, S D=0.30)$ revealed a trend, $t(28)=1.93, \mathrm{p}=0.064, \mathrm{~d}=0.36$. This change in outcome of our inferential statistics is likely due to further reduction in the number of trials included in this comparison. 
Are FOK ratings related to response times during attempts to recall the names corresponding to face cues?

As in Experiment 1, we first investigated whether the response times during recall attempts were related to FOK ratings. Once again, Spearman correlations were computed between these dimensions for individual participants. The mean Spearman correlation between FOK and response time for all trials (Mean $r h o=0.12 \mathrm{SD}=0.19)$ was significantly greater than zero, $\mathrm{t}(28)$ $=3.37, \mathrm{p}=0.002, \mathrm{~d}=0.63$. As in Experiment 1, this significant correlation held even when computed only for trials that lacked perceived recall success (Mean rho $=0.13, \mathrm{SD}=0.21), \mathrm{t}(28)$ $=3.47, \mathrm{p}=0.002, \mathrm{~d}=0.64$. Next, we conducted a $2 \times 2$ ANOVA to assess the influence of priming on response time, and the difference in response times between items later restudied and those not restudied. Focusing on trials without perceived recall success, there was no significant main effect of priming $\mathrm{F}(1,28)=1.76, \mathrm{p}=0.20$, nor a main effect of future restudy decision on response times. However, a significant interaction between these dimensions emerged, such that unprimed, non-studied items, the condition that was also associated with the numerically lowest FOK ratings, had the shortest response times across the four conditions, $F(1,28)=4.91, p=0.04$. Although the lack of main effect of subsequent restudy choices on response times for recall attempts in Experiment 2 diverged from the positive finding we noted in Experiment 1, this result is not entirely surprising given that the range of response times was reduced in Experiment 2 based on alterations in the timing structure of the FOK test phase. Therefore, we feel justified to place more emphasis on our results on response times from Experiment 1 in our overall interpretation. 


\section{Are FOK ratings related to subsequent information-seeking?}

Next, we examined whether the relationship between FOK experiences and subsequent information-seeking behaviour that we observed in Experiment 1 could be replicated even when variability in response times for FOK judgments was limited through restriction of exposure to the face cues. Again, we assessed the relationship of FOK ratings with restudy choices, with data displayed in graphical form in Figure 5A, through the computation of gamma correlations between these variables for individual participants. When this calculation was performed for all trials, the mean gamma correlation was significantly greater than zero $(M=0.41, S D=0.32)$, $t(28)=6.81, p<0.001, d=1.27$, and it remained significantly greater than zero when trials with perceived recall success were excluded from the calculation $(M=0.36, S D=0.27$; note that in the majority of trials, recall was perceived to be unsuccessful, see Table 1 for perceived success frequency), $t(28)=7.17, \mathrm{p}<0.001, d=1.33$ (Figure 5B). Together, these analyses show that FOK ratings remained closely tied to restudy choices, such that cues evoking greater FOK were restudied more often.

\section{Is information-seeking influenced by priming of face cues?}

In order to investigate our second goal of the study, we compared the proportion of face-name pairs with primed face cues that were selected for restudy, with the proportion of pairs with unprimed face cues that were restudied. This comparison closely paralleled how we examined the impact of prior memorisation of face name-pairs on information-seeking behaviour in Experiment 1 but addressed the impact of cue familiarity more directly. Our analysis revealed that participants chose to restudy the names associated with primed faces at a more frequent rate than the names corresponding to unprimed faces (see Table 1 for restudy proportions), $t(28)=$ 4.36, $p<0.001, d=0.81$. This pattern also held when we restricted the analysis to trials in which 
perceived recall was unsuccessful in the FOK test phase (see Table 1 for restudy proportions), $t(28)=4.44, p<0.001, d=0.82$. Taken together these results suggest that cue familiarity, a factor that has previously been shown to influence FOKs in numerous studies (Schwartz and Metcalfe, 1992; Metcalfe et al., 1993; Koriat \& Levy-Sadot, 2001), also influenced subsequent information-seeking behaviour.

\section{Is the relationship between priming and information-seeking tied to FOK ratings?}

The analyses presented on the relationship between priming and subsequent restudy choices so far do not provide information as to whether this effect is tied to FOK ratings or independent. To address this question, a mixed-effects modelling analysis was performed. A similar procedure was used to develop the model as in Experiment 1. Specifically, it included fixed effect terms for FOK rating, cue familiarity and the interaction between these factors (see Supplemental Information Section for details of model development). With this model, we found that restudy choices were predicted by FOK ratings for all items, regardless of the level of familiarity of the cue (see Table 4). For unprimed items, the odds that an item with a given FOK rating would be selected for restudy increased to almost 1.5 times that of an item with a FOK rating 1-point less. For primed items, the odds increased by 1.75 for each FOK rating. The odds, however, were not significantly different for primed as compared to unprimed items, as evident by the nonsignificant interaction between priming and FOK. Finally, there was no significant difference in the odds that highly familiar cues would be restudied compared to those with low familiarity, independent of FOK rating. Overall, these results suggest that the influence of cue familiarity on information-seeking is closely tied to FOK ratings. 


\section{Discussion}

We conducted two experiments that aimed to elucidate links between experiential aspects of memory retrieval and curiosity. Specifically, we examined links between metacognitive FOK experiences and duration of ongoing memory search, subsequent information-seeking, and their relationship. In each experiment we employed an episodic FOK paradigm with face-name pairs that was followed by a restudy phase, which provided means to determine whether FOK experiences bias subsequent information-seeking behaviour towards information that participants could not recall but that they expected to be able to recognise.

Results in Experiment 1 and Experiment 2 replicated the well established positive relationship between the FOK ratings participants provided and corresponding response times. We observed a similar positive relationship between FOK ratings and subsequent informationseeking, as reflected in restudy choices under conditions in which such opportunities were limited. Critically, our experimental manipulations of FOK experiences through alterations in cue familiarity also had parallel effects on information-seeking behaviour in both experiments. In Experiment 1, participants showed higher FOKs for previously studied than novel face cues. This effect on FOKs went hand in hand with longer response times and a bias in subsequent information-seeking for faces that were initially studied compared to those that were novel. In Experiment 2, faces that had been primed prior to initial study were given higher FOK ratings, and were also selected more frequently for subsequent restudy than unprimed faces. Linear mixed-effects modelling revealed that the observed differences in search time (in Experiment 1) and information-seeking behaviour (i.e. away from novel items in Experiment 1 and toward primed items in Experiment 2) that resulted from our experimental manipulations were indeed tied to the effects they exerted on FOK ratings. Overall, these findings suggest that FOK 
experiences at retrieval have pervasive motivational consequences on information-seeking that reflect state curiosity and that can be understood within the theoretical framework of metacognition that emphasise its control function.

The present results replicate and extend the outcome of prior research that has addressed the control function of FOKs as metacognitive experiences. As discussed, numerous studies have reported correlations between FOK experiences and the duration of attempted recall in semantic FOK paradigms (Gruneberg et al., 1977; Nelson \& Narens, 1980; Nelson et al., 1984; Costermans et al., 1992). Experiment 1 showed that this relationship also holds for episodic FOK experiences. Further, past research has also revealed a relationship between FOK experiences and information-seeking behaviour as reflected in restudy choices in an episodic FOK paradigm (Hanczakowski et al., 2014). Notably, however, this relationship was previously demonstrated under conditions in which these choices immediately followed an initial recall attempt for the same item. The current experiments reveal that this motivational consequence of FOK experiences is lasting, and continues to shape information-seeking behaviour even outside of the immediate context of an unsuccessful memory search. Similar to Hanczakowski et al. (2014) the current experiments also show that the impact of cue familiarity, particularly with the more selective priming manipulation in Experiment 2, on FOKs is paralleled by an increase in subsequent restudy choices. Notably, in Experiment 1, the initially studied items that participants chose to seek out more often were the majority of items, while in Experiment 2 participants chose primed items for restudy more frequently and these were the minority of items. This pattern of results across experiments lends support to the conclusion that the manipulation of cue familiarity, rather than the composition of the list, drives the observed biases in information- 
seeking. The mixed-effects models we conducted add to this evidence by suggesting that the experiential aspect of FOKs plays a critical role in the control of behaviour.

Within the rationale of our experiments, we interpret restudy choices as markers of curiosity. Although experiments on the impact of curiosity on learning typically include ratings of curiosity on an item-by-item basis (e.g. Kang et al., 2009; Gruber et al 2014; Metcalfe et al., 2017), we argue that in order to assess curiosity an examination of information-seeking behaviour is sufficient and no additional subjective ratings are required. This argument rests on the definition of curiosity that is widely shared in the literature and makes immediate reference to information seeking behaviour (Loewenstein, 1994; Kidd \& Hayden, 2015; Gottlieb et al., 2016; Metcalfe et al., 2020). Indeed, studies on state curiosity in non-human species and in child development typically adopt this rationale as well (e.g. Smock \& Holt, 1962; Daddaoua,et al., 2016). Moreover, several studies in adult humans on curiosity have also relied on objective behavioural markers, including giving participants a choice as to which stimuli they want to be exposed to (Hsee \& Ruan, 2016; Lau et al., 2018; Oosterwijk et al., 2019), a set-up that parallels the approach we took in the current. Nevertheless, these claims would benefit from further examination in future research on motivational consequences of FOKs, which could include curiosity ratings in the absence of any assessment of restudy behaviour.

Another important consideration, when interpreting our findings in relation to curiosity, is whether the restudy choices participants made were intrinsically motivated, given that this is also a defining characteristic of curiosity. Could it be that participants simply made their restudy choices in a targeted effort to maximize future test performance for the face-name associations (which were probed again in the final phase of the experiment)? To address this point, it is critical to take a closer look at our experimental set-up, and focus on participants' general 
expectations about the goals of the study they participated in. Notably, prior to start, participants were not informed about the structure of the experiment beyond the approximate length and the fact that it is a study on memory and motivation. Other than this general information, they were only given instructions for each phase right at its start. In other words, participants were not informed about the final recognition-memory test until after they had completed the restudy phase. Critically, participants had already performed a memory test (during the FOK test phase) when they entered the restudy phase. With these points in mind, we feel justified in interpreting the restudy behaviour as a reflection of intrinsic motivation in the current study. Future research could provide further evidence to bolster this claim, for example, by including assessment of satisfaction immediately after unveiling of the sought information (see Marvin \& Shohamy, 2016, for a similar approach). If restudy behaviour is reflective of intrinsic motivation, as we argue, then obtaining access to this information can be expected to be experienced as satisfying. Although on the surface decisions to terminate memory search reflect behaviour that is clearly different from decisions that pertain to opportunities for further study, the present results suggest that they may be based on shared motivational mechanisms. Of most relevance, Experiment 1 revealed strong parallels in the effects of the cue-familiarity manipulation on search time and restudy choices, with both effects being tied to FOK experiences. Moreover, items chosen for restudy had longer memory-search durations than those not chosen. While this pattern of results does not establish the presence of shared mechanisms with certainty, they invite this interpretation when considered in the context of work on curiosity. State curiosity is defined in direct relation to information-seeking and is thought to motivate behaviour that resolves uncertainty, with successful access to critical information providing a reward (Kidd \& Hayden, 2015; Gottlieb et al., 2016; Gottlieb \& Oudeyer 2018; Gruber \& 
Ranganath, 2019). Although curiosity is typically defined with reference to exploration of the external environment in an attempt to acquire information or knowledge (Berlyne, 1966; Gottlieb et al., 2013), such a definition could also be applied to 'internal' memory search. In a nutshell, memory search also involves information-seeking that aims to resolve uncertainty. Metacognitive retrieval experiences that arise during this search may trigger motivational mechanisms that could drive ongoing retrieval efforts as well as future behaviour geared towards further exploration of the external environment. Future research may build on this curiositybased framework so as to identify the suggested shared motivational mechanisms. For example, future imaging studies could determine whether the engagement of reward circuitry predicts both types of information-seeking behaviour.

Other findings from research on metacognition indicate that the reported links between retrieval experiences and curiosity may not be limited to FOKs. Indeed a similar relationship has been documented for the TOT phenomenon (Litman et al., 2005; Metcalfe et al., 2017). Litman et al. (2005) presented participants with general knowledge questions and asked them to indicate whether they knew or did not know the answer, or whether they were in a TOT state (i.e. "The answer is on the tip-of-my-tongue"). Following these questions, participants provided a curiosity rating for each fact and, in a final phase of the experiment, they were allowed to explore the answers to any of the questions that had been presented earlier. Results showed that facts which induced a TOT experience received higher curiosity ratings and were more frequently explored, relative to those participants knew or did not know. Metcalfe et al. (2017) obtained similar findings when they probed information-seeking behaviour immediately following a TOT experience for semantic facts. 
Research on judgements-of-learning (JOL) suggests that a positive relationship between metacognitive experiences and curiosity is, however, not ubiquitous across all metacognitive judgements. DeCaro \& Thomas (2019) had participants attempt to recall members of previously studied word-pairs, using the other pair-members as cues. Following this recall attempt, participants provided a JOL, which probes the likelihood a word-pair could be successfully learned during a future study phase. The experiment also included a restudy phase that required participants to select a subset of items for further memorisation. Results revealed a significant correlation between reported JOL experiences and restudy choices. Unlike in the current study and in research on TOTs, however, the correlation between metacognitive ratings and restudy choices was found to be negative, such that items with lower JOL ratings were restudied more frequently than those with higher ratings. This pattern of findings across studies raises the interesting question as to what component-processes trigger the motivational mechanisms that increase subsequent information-seeking behaviour. This question deserves careful consideration in future research involving the examination of information-seeking following systematic manipulation of different types of memory judgments.

Within the broader literature on curiosity, the current findings can be interpreted in relation to Loewenstein's influential theory on information gaps. This work suggests that curiosity arises due to differences between what we want to know and what we actually know, a factor termed an information gap (Loewenstein, 1994). In other words, from Loewenstein's perspective, for curiosity to be initiated there must be an unknown piece of information, and the individual must actually want to know about this information. Importantly however, recent work has argued that, while information gaps are indeed critical, for curiosity to be highest, the gap may need to be in a specific range. This optimal information gap has been deemed the Region of 
Proximal Learning (RPL), and can be defined as the situation where an information gap is small enough to be judged as possible to be closed (Metcalfe \& Kornell, 2003; Metcalfe \& Kornell, 2005; Metcalfe et al., 2017; Metcalfe et al., 2020). Put another way, the gap must not be so large that individuals feel they will not learn the information, but also not so small that they perceive they have more or less mastered it. A recent review by Metcalfe and colleagues suggests a important link between the RPL and curiosity (2020). In their framework, the two critical steps to spark curiosity are an unsuccessful recall attempt and a metacognitive experience indicating that the sought-after information is close to being learned, or within the RPL. The metacognitive retrieval experience most frequently examined in studies on the RPL is the TOT state (e.g. Metcalfe et al., 2017; Bloom et al., 2018; see DeCaro et al., 2019 for pertinent research on JOLs), as it intuitively reflects an experience in which information can almost but not completely be accessed. Considered in the context of the current study, we propose that FOK experiences also fulfill the two steps of the RPL that spark curiosity. Specifically, we report that these experiences predicted restudy choices under conditions in which recall was perceived to be unsuccessful, and in which participants had the impression that they could easily recognize the corresponding name. From this perspective, our observation that participants' tendency to restudy items that induced high FOKs is in line with the idea that curiosity peaks when the information gap for an item is in the RPL.

While we provide evidence in support of a relationship between FOK experiences and the control of behaviour as reflected in response time during memory search and in subsequent restudy choices, we recognise that it remains difficult to establish causality in this observed relationship. Notably, it has been suggested that response times may not be the consequence of FOKs but rather a heuristic clue that informs them (see Koriat, 2007 and Metcalfe, 2009 for 
discussion). For restudy choices, concerns about cause and effect may be less pressing in the current study, given that they followed the expression of FOKs in a separate experimental phase. The results of our experimental manipulation of FOKs provides additional evidence that gives credence to a causal interpretation, again particularly for information-seeking behaviour during restudy. By virtue of introducing entirely novel (Experiment 1) or primed faces (Experiment 2) in the FOK test phase, we were able to decrease or increase FOKs in a predictable manner, respectively, and influence information-seeking in a parallel fashion. Definitive evidence for a casual role could be established through direct manipulations of the neural mechanisms that drive information-seeking behaviour. As noted, interactions between brain regions that form the reward circuitry are closely tied to curiosity and involve dopamine as their primary neurotransmitter (see Gruber \& Ranganath, 2019, for review). More specifically, studies have shown these reward-related areas track the degree of self-reported curiosity (e.g. Kang et al., 2009; Gruber et al., 2014). As such, pharmacological manipulations of dopamine may allow for the assessment of a causal relationship between FOKs and the objective markers of curiosity probed in the current study. A related prediction is that the pharmacological alteration of dopamine (e.g. through the dopamine D2 receptor antagonist haloperidol) would lead to a decoupling between FOKs, search times, and subsequent restudy choice behaviour (see Clos et al., 2019, for a suitable study design).

In conclusion, the present study revealed that curiosity is not only intimately tied to learning but also has links to episodic memory retrieval. The evidence presented argues in favour of the general notion that metacognitive experiences accompanying unsuccessful retrieval from episodic memory can induce states of curiosity that exert control over information-seeking behaviour beyond the immediate retrieval context. From this perspective, curiosity may act as a 
bond that ensures that memory gaps identified through unsuccessful retrieval can adaptively guide future learning.

\section{Acknowledgements:}

We would like to thank Hanna Wagner for help with data collection and Renée DeCaro for sharing scripts for linear mixed-effects models.

\section{Disclosure Statement}

No potential conflict of interest was reported by the authors.

\section{Funding}

This work was supported by the Natural Sciences and Engineering Research Council of Canada under a Discovery Grant [RGPIN 5770] to SK and an Alexander Graham Bell Canadian Graduate Scholarship Masters to GB.

\section{References}

Berlyne, D. E. (1966). Curiosity and exploration. Science, 153(3751), 25-33.

Bloom, P. A., Friedman, D., Xu, J., Vuorre, M., \& Metcalfe, J. (2018). Tip-of-the-tongue states predict enhanced feedback processing and subsequent memory. Consciousness and Cognition, 63, 206-217. https://doi.org/10.1016/j.concog.2018.05.010

Brown, R., \& McNeill, D. (1966). The "tip of the tongue" phenomenon. Journal of Verbal Learning and Verbal Behavior, 5(4), 325-337. https://doi.org/10.1016/S00225371(66)80040-3

Clos, M., Bunzeck, N., \& Sommer, T. (2019). Dopamine is a double-edged sword: dopaminergic modulation enhances memory retrieval performance but impairs metacognition. Neuropsychopharmacology, 44(3), 555-563. https://doi.org/10.1038/s41386-018-0246-y

Costermans, J., Lories, G., \& Ansay, C. (1992). Confidence Level and Feeling of Knowing in Question Answering: The Weight of Inferential Processes. Journal of Experimental Psychology: Learning, Memory, and Cognition, 18(1), 142-150. https://doi.org/10.1037/0278-7393.18.1.142 
Daddaoua, N., Lopes, M., \& Gottlieb, J. (2016). Intrinsically motivated oculomotor exploration guided by uncertainty reduction and conditioned reinforcement in non-human primates. Scientific Reports, 6(December 2015), 1-15. https://doi.org/10.1038/srep20202

DeCaro, R., \& Thomas, A. K. (2019). How attributes and cues made accessible through monitoring affect self-regulated learning in older and younger adults. Journal of Memory and Language, 107, 69-79. https://doi.org/https://doi.org/10.1016/j.jml.2019.04.002

Fastrich, G. M., Kerr, T., Castel, A. D., \& Murayama, K. (2018). The role of interest in memory for trivia questions: An investigation with a large-scale database. Motivation Science, 4(3), 227-250. https://doi.org/10.1016/j.physbeh.2017.03.040

Fiacconi, C. M., Kouptsova, J. E., \& Köhler, S. (2017). A role for visceral feedback and interoception in feelings-of-knowing. Consciousness and Cognition, 53, 70-80. https://doi.org/http://dx.doi.org/10.1016/j.concog.2017.06.001

Gottlieb, J., Lopes, M., \& Oudeyer, P. Y. (2016). Motivated cognition: neural and computational mechanisms of curiosity, attention and intrinsic motivation. In S. Kim, J. Reeve, \& M. Bong. (Eds.), Recent Developments in Neuroscience Research on Human Motivation (Advances in Motivation and Achievement) (pp. 149-172). Emerald Group Publishing Limited. https://doi.org/https://doi.org/10.1108/S0749-742320160000019017

Gottlieb, J., \& Oudeyer, P. Y. (2018). Towards a neuroscience of active sampling and curiosity. Nature Reviews Neuroscience, 19(12), 758-770. https://doi.org/10.1038/s41583-0180078-0

Gottlieb, J., Oudeyer, P. Y., Lopes, M., \& Baranes, A. (2013). Information-seeking, curiosity, and attention: Computational and neural mechanisms. Trends in Cognitive Sciences, 17(11), 585-593. https://doi.org/10.1016/j.tics.2013.09.001

Gruber, M. J., Gelman, B. D., \& Ranganath, C. (2014). States of Curiosity Modulate Hippocampus-Dependent Learning via the Dopaminergic Circuit. Neuron, 84(2), 486496. https://doi.org/10.1016/j.neuron.2014.08.060

Gruber, M. J., \& Ranganath, C. (2019). How Curiosity Enhances Hippocampus-Dependent Memory: The Prediction, Appraisal, Curiosity, and Exploration (PACE) Framework. Trends in Cognitive Sciences, 23(12), 1014-1025. https://doi.org/https://doi.org/10.1016/j.tics.2019.10.003 
Gruneberg, M. M., Monks, J., \& Sykes, R. N. (1977). Some methodological problems with feeling of knowing studies. Acta Psychologica, 41, 365-371. https://doi.org/10.1016/0001-6918(77)90014-2

Hanczakowski, M., Zawadzka, K., \& Cockcroft-McKay, C. (2014). Feeling of knowing and restudy choices. Psychonomic Bulletin and Review, 21(6), 1617-1622. https://doi.org/10.3758/s13423-014-0619-0

Hart, J. T. (1965). Memory and the feeling-of-knowing experience. Journal of Educational Psychology, 56(4), 208-216. http://arxiv.org/abs/1012.0819

Hosey, L. A., Peynircioğlu, Z. F., \& Rabinovitz, B. E. (2009). Feeling of knowing for names in response to faces. Acta Psychologica, 130(3), 214-224. https://doi.org/10.1016/j.actpsy.2008.12.007

Hsee, C. K., \& Ruan, B. (2016). The Pandora Effect: The Power and Peril of Curiosity. Psychological Science, 27(5), 659-666. https://doi.org/10.1177/0956797616631733

Isingrini, M., Sacher, M., Perrotin, A., Taconnat, L., Souchay, C., Stoehr, H., \& Bouazzaoui, B. (2016). Episodic feeling-of-knowing relies on noncriterial recollection and familiarity: Evidence using an online remember-know procedure. Consciousness and Cognition, 41, 31-40. https://doi.org/10.1016/j.concog.2016.01.011

Kang, M. J., Hsu, M., Krajbich, I. M., Loewenstein, G., McClure, S. M., Wang, J. T., \& Camerer, C. F. (2009). The Wick in the Candle of Learning: Epistemic Curiosity Activates Reward Circuitry and Enhances Memory. Psychological Science, 20(8), 963973. https://doi.org/10.1111/j.1467-9280.2009.02402.x

Kidd, C., \& Hayden, B. Y. (2015). The Psychology and Neuroscience of Curiosity. Neuron, 88(3), 449-460. https://doi.org/http://dx.doi.org/10.1016/j.neuron.2015.09.010

Koriat, A. (2007). Metacognition and Consciousness. In P. D. Zelazo, M. Moscovitch, \& E. Thompson (Eds.), The Cambridge Handbook of Consciousness (pp. 289-325). Cambridge University Press.

Koriat, A. (1993). How do we know that we know? The accessibility model of the feeling of knowing. Psychological Review, 100(4), 609-639. https://doi.org/10.1037/0033295X.100.4.609

Koriat, A., \& Levy-Sadot, R. (2001). The Combined Contributions of the Cue-Familiarity and Accessibility Heuristics to Feelings of Knowing. Journal of Experimental Psychology: 
Learning Memory and Cognition, 27(1), 34-53. https://doi.org/10.1037/02787393.27.1.34

Lau, J. K. L., Ozono, H., Kuratomi, K., Komiya, A., \& Murayama, K. (2020). Shared striatal activity in decisions to satisfy curiosity and hunger at the risk of electric shocks. Nature Human Behaviour, 4(5), 531-543. https://doi.org/10.1038/s41562-020-0848-3

Litman, J. A., Hutchins, T. L., \& Russon, R. K. (2005). Epistemic curiosity, feeling-of-knowing, and exploratory behaviour. Cognition and Emotion, 19(4), 559-582. https://doi.org/10.1080/02699930441000427

Loewenstein, G. (1994). The psychology of curiosity: a review and reinterpretation. Pyschological Bulletin, 116(1), 75-98. https://doi.org/10.1080/02699930441000427

Ma, D. S., Correll, J., \& Wittenbrink, B. (2015). The Chicago face database: A free stimulus set of faces and norming data. Behavior Research Methods, 47(4), 1122-1135. https://doi.org/10.3758/s13428-014-0532-5

Marvin, C. B., \& Shohamy, D. (2016). Curiosity and reward: Valence predicts choice and information prediction errors enhance learning. Journal of Experimental Psychology: General, 145(3), 266-272. https://doi.org/10.1037/xge0000140

McGillivray, S., Murayama, K., \& Castel, A. D. (2015). Thirst for knowledge: The effects of curiosity and interest on memory in younger and older adults. Psychology and Aging, 30(4), 835-841. https://doi.org/doi:10.1037/a0039801

Metcalfe, J. (2009). Metacognitive judgments and control of study. Current Directions in Psychological Science, 18(3), 159-163. https://doi.org/10.1111/j.14678721.2009.01628.x

Metcalfe, J., \& Finn, B. (2008). Evidence that judgments of learning are causally related to study choice. Psychonomic Bulletin and Review, 15(1), 174-179. https://doi.org/10.3758/PBR.15.1.174

Metcalfe, J., \& Kornell, N. (2003). The Dynamics of Learning and Allocation of Study Time to a Region of Proximal Learning. Journal of Experimental Psychology: General, 132(4), 530-542. https://doi.org/10.1037/0096-3445.132.4.530

Metcalfe, J., \& Kornell, N. (2005). A Region of Proximal Learning model of study time allocation. Journal of Memory and Language, 52(4), 463-477. https://doi.org/10.1016/j.jml.2004.12.001 
Metcalfe, J., Schwartz, B. L., \& Bloom, P. A. (2017). The tip-of-the-tongue state and curiosity. Cognitive Research: Principles and Implications, 2(1), 1-8. https://doi.org/10.1186/s41235-017-0065-4

Metcalfe, J., Schwartz, B. L., \& Eich, T. S. (2020). Epistemic curiosity and the region of proximal learning. Current Opinion in Behavioral Sciences, 35, 40-47. https://doi.org/10.1016/j.cobeha.2020.06.007

Metcalfe, J., Schwartz, B. L., \& Joaquim, S. G. (1993). The Cue-Familiarity Heuristic in Metacognition. Journal of Experimental Psychology: Learning, Memory, and Cognition, 19(4), 851-861. https://doi.org/10.1037/0278-7393.19.4.851

Moulin, C. J. A., \& Souchay, C. (2014). Epistemic feelings and memory. In T. J. Perfect \& D. S. Lindsay (Eds.), The SAGE Handbook of Applied Memory (pp. 520-538). SAGE Publications Ltd. https://doi.org/10.4135/9781446294703

Nelson, T. O. (1984). A comparison of current measures of the accuracy of feeling-of-knowing predictions. Psychological Bulletin, 95(1), 109-133. https://doi.org/10.1037//00332909.95.1.109

Nelson, T. O. (1996). Consciousness and metacognition. American Psychologist, 51(2), $102-$ 116. https://doi.org/10.1037//0003-066x.51.2.102Nelson, T. O., Gerler, D., \& Narens, L. (1984). Accuracy of feeling-of-knowing judgments for predicting perceptual identification and relearning. Journal of Experimental Psychology: General, 113(2), 282300. https://doi.org/10.1037/0096-3445.113.2.282

Nelson, T. O., \& Leonesio, R. J. (1988). Allocation of Self-Paced Study Time and the "Labor-inVain Effect." Journal of Experimental Psychology: Learning, Memory, and Cognition, 14(4), 676-686. https://doi.org/10.1037/0278-7393.14.4.676Nelson, T. O., \& Narens, L. (1980). Norms of 300 general-information questions: Accuracy of recall, latency of recall, and feeling-of-knowing ratings. Journal of Verbal Learning and Verbal Behavior, 19(3), 338-368. https://doi.org/10.1016/S0022-5371(80)90266-2

Nelson, T. O., \& Narens, L. (1990). Metamemory: A Theoretical Framework and New Findings. Psychology of Learning and Motivation - Advances in Research and Theory, 26, 125173. https://doi.org/10.1016/S0079-7421(08)60053-5 
Nelson, T., \& Narens, L. (1996). Why Investigate Metacognition? In J. Metcalfe \& A. P. Shimamura (Eds.), Metacognition: Knowing about Knowing (pp. 1-26). MIT Press. https://doi.org/10.7551/mitpress/4561.003.0003

Oosterwijk, S., Snoek, L., Tekoppele, J., Engelbert, L. H., \& Scholte, H. S. (2020). Choosing to view morbid information involves reward circuitry. Scientific Reports, 10(1), 1-13. https://doi.org/10.1038/s41598-020-71662-y

Reder, L. M. (1987). Strategy selection in question answering. Cognitive Psychology, 19, 90138. https://doi.org/10.1016/0010-0285(87)90005-3

Reder, L. M., \& Ritter, F. E. (1992). What Determines Initial Feeling of Knowing? Familiarity With Question Terms, Not With the Answer. Journal of Experimental Psychology: Learning, Memory, and Cognition, 18(3), 435-451. https://doi.org/10.1037/02787393.18.3.435

Schwartz, B. L. (2001). The relation of tip-of-the-tongue states and retrieval time. Memory and Cognition, 29(1), 117-126. https://doi.org/10.3758/BF03195746

Schwartz, B. L., \& Metcalfe, J. (1992). Cue Familiarity but not Target Retrievability Enhances Feeling-of-Knowing Judgments. Journal of Experimental Psychology: Learning, Memory, and Cognition, 18(5), 1074-1083. https://doi.org/10.1037/0278-7393.18.5.1074

Schwartz, B. L., Pillot, M., \& Bacon, E. (2014). Contextual information influences the feeling of knowing in episodic memory. Consciousness and Cognition, 29, 96-104. https://doi.org/10.1016/j.concog.2014.08.018

Singer, M., \& Tiede, H. L. (2008). Feeling of knowing and duration of unsuccessful memory search. Memory and Cognition, 36(3), 588-597. https://doi.org/10.3758/MC.36.3.588

Smock, C. D., \& Holt, B.-G. (1962). Children's Reactions to Novelty: An Experimental Study of "Curiosity Motivation." Child Development, 33(3), 631-642.

Stare, C. J., Gruber, M. J., Nadel, L., Ranganath, C., \& Gómez, R. L. (2018). Curiosity-driven memory enhancement persists over time but does not benefit from post-learning sleep. Cognitive Neuroscience, 9(3-4), 100-115. https://doi.org/10.1080/17588928.2018.1513399

Thomas, A. K., Bulevich, J. B., \& Dubois, S. J. (2011). Context Affects Feeling-of-Knowing Accuracy in Younger and Older Adults. Journal of Experimental Psychology: Learning Memory and Cognition, 37(1), 96-108. https://doi.org/10.1037/a0021612 
Table 1.

Summary of behavioural data for Experiments 1 and 2.

\section{Experiment 1}

\begin{tabular}{lllll}
\hline & & $\begin{array}{l}\text { No Perceived } \\
\text { Recall Success }\end{array}$ & $\begin{array}{l}\text { Perceived } \\
\text { Recall Success }\end{array}$ & All Trials \\
\hline Previously & Proportion of trials & $0.92(0.12)$ & $0.08(0.12)$ & \\
Studied & FOK rating & $2.26(0.49)$ & $4.51(0.65)$ & $2.42(0.51)$ \\
& Proportion restudied & $0.48(0.10)$ & $0.68(0.38)$ & $0.49(0.09)$ \\
& Recognition accuracy & $0.47(0.12)$ & $0.56(0.36)$ & $0.48(0.12)$ \\
& Response time (ms) & $3386(2032)$ & $5106(3155)$ & $3410(1953)$ \\
Novel & Proportion of trials & $0.98(0.05)$ & $0.02(0.05)$ & \\
& FOK rating & $1.54(0.36)$ & $4.36(0.72)$ & $1.59(0.41)$ \\
& Proportion restudied & $0.41(0.15)$ & $0.59(0.45)$ & $0.41(0.14)$ \\
& Recognition accuracy & $0.36(0.12)$ & $0(0)$ & $0.36(0.12)$ \\
Total & Response time (ms) & $3019(1927)$ & $4928(4100)$ & $3028(1918)$ \\
\hline
\end{tabular}

Experiment 2

\begin{tabular}{lllll}
\hline & & $\begin{array}{l}\text { No Perceived } \\
\text { Recall Success }\end{array}$ & $\begin{array}{l}\text { Perceived } \\
\text { Recall Success }\end{array}$ & All Trials \\
\hline Primed & Proportion of trials & $0.76(0.17)$ & $0.24(0.17)$ & \\
& FOK rating & $2.68(0.48)$ & $4.30(0.53)$ & $3.08(0.45)$ \\
& Proportion restudied & $0.50(0.11)$ & $0.67(0.26)$ & $0.54(0.10)$ \\
& Recognition accuracy & $0.45(0.18)$ & $0.58(0.43)$ & $0.47(0.18)$ \\
Unprimed & Proportion of trials & $0.86(0.11)$ & $0.14(0.11)$ & \\
& FOK rating & $2.24(0.34)$ & $4.26(0.52)$ & $2.49(0.35)$ \\
& Proportion restudied & $0.38(0.11)$ & $0.67(0.28)$ & $0.42(0.09)$ \\
& Recognition accuracy & $0.48(0.14)$ & $0.70(0.33)$ & $0.49(0.14)$ \\
Total & Proportion of trials & $0.83(0.12)$ & $0.17(0.12)$ & \\
\hline
\end{tabular}

Note: Data are shown as Mean (SD). Recognition accuracy is for trials not selected for restudy 
Table 2.

Results of the mixed-effects model used to predict the response times during the FOK test phase in Experiment 1.

\begin{tabular}{|c|c|c|c|c|}
\hline \multicolumn{2}{|l|}{ Fixed Effects } & $\beta(\mathrm{SE})$ & $t$ & $p$ \\
\hline \multicolumn{2}{|l|}{ Intercept } & $0.59(0.13)$ & 4.74 & $<0.001$ \\
\hline \multicolumn{2}{|l|}{ FOK } & $0.23(0.04)$ & 6.47 & $<0.001$ \\
\hline \multicolumn{2}{|c|}{ Cue Status } & $0.07(0.06)$ & 1.21 & 0.23 \\
\hline \multicolumn{2}{|c|}{ FOK x Status (Initially Studied) } & $-0.05(0.03)$ & -1.73 & 0.08 \\
\hline \multicolumn{2}{|c|}{ Random Effects } & \multicolumn{3}{|c|}{ Variance (SD) } \\
\hline \multirow[t]{2}{*}{ Intercept } & Item & $0.01(0.03)$ & & \\
\hline & Subject & $0.24(0.49)$ & & \\
\hline Slope - Item & FOK & $0.00(0.03)$ & & \\
\hline Slope - Subject & FOK & $0.02(0.12)$ & & \\
\hline Residual & & $0.45(0.67)$ & & \\
\hline
\end{tabular}


Table 3 .

Results of the mixed-effects model used to predict subsequent information-seeking choices in Experiment 1.

\begin{tabular}{|c|c|c|c|c|}
\hline \multicolumn{2}{|l|}{ Fixed Effects } & $\beta(\mathrm{SE})$ & $z$ & $p$ \\
\hline \multicolumn{2}{|l|}{ Intercept } & $-0.61(0.19)$ & -3.20 & 0.0014 \\
\hline \multicolumn{2}{|l|}{ FOK } & $0.14(0.095)$ & 1.43 & 0.15 \\
\hline \multicolumn{2}{|l|}{ Cue Status } & $-0.24(0.21)$ & -1.12 & 0.26 \\
\hline \multicolumn{2}{|c|}{ FOK x Status (Initially Studied) } & $0.22(0.10)$ & 2.16 & 0.030 \\
\hline \multicolumn{2}{|l|}{ Random Effects } & \multicolumn{3}{|l|}{ Variance (SD) } \\
\hline Intercept & Item & $0.013(0.11)$ & & \\
\hline \multirow[t]{3}{*}{ Slope - Item } & Cue Status (Novel) & $0.014(0.12)$ & & \\
\hline & Cue Status (Initially Studied) & $0.095(0.31)$ & & \\
\hline & FOK & $0.00(0.00)$ & & \\
\hline \multirow[t]{3}{*}{ Slope - Subject } & Cue Status (Novel) & $0.38(0.62)$ & & \\
\hline & Cue Status (Initially Studied) & $0.091(0.30)$ & & \\
\hline & FOK & $0.025(0.16)$ & & \\
\hline
\end{tabular}


Table 4.

Results of the mixed-effects model used to predict subsequent information-seeking choices in Experiment 2.

\begin{tabular}{|c|c|c|c|c|}
\hline \multicolumn{2}{|l|}{ Fixed Effects } & $\beta(\mathrm{SE})$ & $z$ & $p$ \\
\hline \multicolumn{2}{|l|}{ Intercept } & $-1.39(0.18)$ & -7.86 & $<0.001$ \\
\hline \multicolumn{2}{|l|}{ FOK } & $0.38(0.065)$ & 5.75 & $<0.001$ \\
\hline \multicolumn{2}{|l|}{ Cue Status } & $-0.058(0.30)$ & -0.20 & 0.84 \\
\hline \multicolumn{2}{|c|}{ FOK x Status (Primed) } & $0.18(0.11)$ & 1.65 & 0.010 \\
\hline \multicolumn{2}{|l|}{ Random Effects } & \multicolumn{3}{|l|}{ Variance (SD) } \\
\hline Intercept & Item & $0.0056(0.075)$ & & \\
\hline \multirow[t]{3}{*}{ Slope - Item } & Cue Status (Unprimed) & $0.040(0.20)$ & & \\
\hline & Cue Status (Primed) & $0.031(0.18)$ & & \\
\hline & FOK & $0.022(0.15)$ & & \\
\hline \multirow[t]{3}{*}{ Slope - Subject } & Cue Status (Unprimed) & $0.19(0.44)$ & & \\
\hline & Cue Status (Primed) & $0.060(0.25)$ & & \\
\hline & FOK & $0.00(0.00)$ & & \\
\hline
\end{tabular}

